\title{
A Remark on Gupta, Gupta and Singh Optional Randomized Response Model
}

\author{
H. P. SINGH AND S. M. GOREY
}

\begin{abstract}
Gupta et al (2002) suggested an optional randomized response model under the assumption that the mean of the scrambling variable $S$ is 'unity' [i.e. $\mu_{s}=1$ ]. This assumption limits the use of Gupta et al's (2002) randomized response model. Keeping this in view we have suggested a modified optional randomized response model which can be used in practice without any supposition and restriction over the mean $\left(\mu_{s}\right)$ of the scrambling variables $S$. It has been shown that the estimator of the mean of the stigmatized variable based on the proposed optional randomized response sampling is more efficient than the Eicchorn and Hayre (1983) procedure and Gupta et al's (2002) optional randomized technique when the mean of the scrambling $S$ is larger than unity [i.e. $\mu_{s}>1$ ]. A numerical illustration is given in support of the present study.
\end{abstract}

Mathematics Subject Classification 2010: 62D05.

Keywords: Empirical study, Mean, Optional randomization response technique, Sensitivity level, Variance.

\section{INTRODUCTION}

The problem of estimating the population mean of a sensitive quantitative variable is well recognized in survey sampling. It is easier to get responses to nonsensitive questions than to personal sensitive questions. It may happen due to the involvement of controversial assertions, stigmatizing and/or incriminating matters which people like to hide, for reasons of modesty, fear of being thought bigoted, or merely a reluctance to confide secretly to a stranger. Warner (1965) was the first to introduce an ingenuous procedure to estimate the incidence of attributes of sensitive nature such as induced abortions, a drug used etc. through a randomization device. A rich growth of literature on randomized response techniques can be found in Tracy and Mangat (1996), Zou(1997), Singh and Joarder (1997), Bhargava and Singh (2001,2002), Singh and Mathur (2003), Gjestvang and Singh (2006), Singh and Tarray (2014), Tarray et al (2015), Tarray and Singh (2017). 
Eichhorn and Hayre (1983) proposed a scrambled randomized response method for estimating the mean $\mu_{x}$ and the variance $\sigma_{x}^{2}$ of the sensitive quantitative variable, say $X$. Following them, each respondent selected in the sample is instructed to use a randomization device and generate a random number, say $S$, from some preassigned distribution. The distribution of the scrambling variable $S$ is assumed to be known. The mean $\mu_{s}$ and variance $\gamma_{s}^{2}$ of the scrambling variable $S$ are known. The ith respondent selected in the sample of size $n$, drawn by using simple random sampling with replacement (SRSWR), is requested to report the value $Z=\frac{S X_{i}}{\theta}$ as a scrambled response on the sensitive variable $X$. Eichhorn and Hayre (1983) suggested an unbiased estimator of the population mean $\mu_{x}$ of $X$, as

$$
\hat{\mu}_{x(E H)}=\frac{1}{n} \sum_{i=1}^{n} Z_{i}
$$

with variance

$$
\operatorname{Var}\left(\hat{\mu}_{x(E H)}\right)=(1 / n)\left[\sigma_{x}^{2}+C_{\gamma}^{2}\left(\sigma_{x}^{2}+\mu_{x}^{2}\right)\right]
$$

where $C_{\gamma}=\gamma_{s} / \mu_{s}$ denotes the known coefficient of variation of the scrambling variable $S$.

\section{- Gupta et al (2002) Optional Randomization Procedure}

In Gupta et al (2002) optional randomized response technique, each respondent selected in the SRSWR sample is instructed to use a randomization device and generate a positive-valued random number $S$ from a given probability distribution with known $\mu_{s} \cong 1$ and have known $\gamma_{s}^{2}$. Then he or she is requested to report one of the following questions:

(a) the correct response $X$, or

(b) the scrambled response $S X$, which is determined by the respondent himself. 
The optional randomized response model can then be written as

$$
Z=S^{Y} X
$$

where $Y$ is a random variable defined as

$$
Y=\left\{\begin{array}{l}
1, \text { if the response is scrambled } \\
0, \text { otherwise }
\end{array}\right.
$$

It is to be noted that $Y$ is a Bernoulli variable with $E(Y)=w$, where $w$ is the probability that a person will report the scrambled response rather than the actual response $X$. Here $w$ is known as sensitivity level. If a question in the survey is more sensitive than more people will report scrambled responses and the value of $w$ will be close to 1 . If the question is not very sensitive, then the value of $w$ will be close to ' 0 '. Thus $w$ is a measure of the level of sensitivity of the question in the personal interview surveys.

Following this device, Gupta et al (2002) suggested an unbiased estimator for population mean $\mu_{x}$ of the sensitive characteristics $X$ as

$$
\hat{\mu}_{x(G)}=\frac{1}{n} \sum_{i=1}^{n} Z_{i}
$$

whose variance is given by

$$
V\left(\hat{\mu}_{x(G)}\right)=\frac{1}{n}\left[\sigma_{x}^{2}+w \gamma_{s}^{2}\left(\sigma_{x}^{2}+\mu_{x}^{2}\right)\right]
$$

Gupta et al (2002) have further suggested an estimator of the sensitivity level $w$ as

$$
\hat{w}_{G}=\frac{\left[\frac{1}{n} \sum_{i=1}^{n} \log \left(Z_{i}\right)-\log \left(\frac{1}{n} \sum_{i=1}^{n} Z_{i}\right)\right]}{E[\log S]}
$$


- Logical Reason Behind Assumption of $\mu_{s}=1$ in Gupta et al (2002) Optional Randomized Response Model

In Gupta et al (2002) procedure it is assumed that the value of the mean $\mu_{s}$ of the scrambled variable $S$ is unity (i.e. $\mu_{s}=1$ ). Thereby meaning is that the optional randomized response model due to Gupta et al (2002) will work for $\mu_{s}=1$.

In Gupta et al (2002) model, the expected value of the observed response $Z$ is given by

$$
\begin{aligned}
E(Z) & =E\left[S^{Y} X\right] \\
& =E\left[S^{Y} X \mid Y=1\right] P(Y=1)+E\left[S^{Y} X \mid Y=0\right] P(Y=0) \\
& =E(S X) P(Y=1)+E(X) P(Y=0) \\
& =E(S) E(X) P(Y=1)+E(X) P(Y=0) \\
& =\mu_{s} \mu_{x} w+\mu_{x}(1-w) \\
& =\mu_{x}\left[\mu_{s} w+(1-w)\right] \\
& =\mu_{x}\left[w\left(\mu_{s}-1\right)+1\right]
\end{aligned}
$$

It is obvious from (1.7) that $E(Z)=\mu_{x}$ only when $\mu_{s}=1$. Unless $\mu_{s}=1$, the estimator $\mu_{x(G)}$ proposed by Gupta et al (2002) cannot be unbiased. So to obtain the unbiased estimator of the mean $\mu_{x}$ through their randomized response technique Gupta et al (2002) assumed that $\mu_{s}=1$.

Now a question arises that what will happen if $\mu_{s} \neq 1$ ? So the optional randomized response model due to Gupta et al (2002) needs modification so that the modified randomized response model holds in the situation, where $\mu_{s} \neq 1$. Keeping this in view we have suggested a modified optional randomized response model and studied its properties. 


\section{PROPOSED OPTIONAL RANDOMIZED RESPONSE MODEL}

It is known that the distribution of the scrambling variable $S$ is known (i.e. the mean $\mu_{s}$ and variance $\gamma_{s}^{2}$ of the scrambling variable $S$ are known). Thus, using the known value of mean $\mu_{s}$, we have suggested a modified optional randomized procedure, each respondent selected in the SRSWR sample chosen one of the following two options:

(i) the respondent can report the correct response, or

(ii) the respondent can report the scrambled response $\frac{S X}{\mu_{s}}$.

Here we have assumed that both $S$ and $X$ are positive valued random variables. Thus the proposed optional randomized response model can be written as

$$
Z=\left(\frac{S}{\mu_{s}}\right)^{Y} X
$$

where $Y=1$ or $O$ according to as the response is scrambled or not.

Following the proposed optional randomized response device we state the following theorems.

THEOREM 1. An unbiased estimator of the population mean $\mu_{x}$ is given by

$$
\hat{\mu}_{x(S G)}=\frac{1}{n} \sum_{i=1}^{n} Z_{i}
$$

PROOF. Taking expectation of both sides of (2.2) we have

$$
\begin{aligned}
E\left(\hat{\mu}_{x(S G)}\right) & =\frac{1}{n} \sum_{i=1}^{n} E\left(Z_{i}\right) \\
& =\frac{1}{n} \sum_{i=1}^{n}\left[E\left\{\left(\frac{S}{\mu_{s}}\right)^{Y} X_{i} \mid Y=1\right\} P(Y=1)\right.
\end{aligned}
$$




$$
\begin{aligned}
& =\frac{1}{n} \sum_{i=1}^{n}\left[E\left(\frac{S X_{i}}{\mu_{s}}\right) P(Y=1)+E\left(X_{i}\right) P(Y=0)\right] \\
& \left.\quad+E\left\{\left(\frac{S}{\mu_{s}}\right)^{Y} X_{i} \mid Y=0\right\} P(Y=0)\right] \\
& =\frac{1}{n} \sum_{i=1}^{n}\left[\frac{E(S) E\left(X_{i}\right)}{\mu_{s}} P(Y=1)+E\left(X_{i}\right) P(Y=0)\right] \\
& =\frac{1}{n} \sum_{i=1}^{n}\left[\frac{\mu_{s} \mu_{x}}{\mu_{s}} w+\mu_{x}(1-w)\right] \\
& =\frac{1}{n} \sum_{i=1}^{n}\left[\mu_{x} w+\mu_{x}(1-w)\right] \\
& =\frac{1}{n} \sum_{i=1}^{n} \mu_{x}=\mu_{x}
\end{aligned}
$$

which proves the theorem.

THEOREM 2. The variance of the proposed estimator $\hat{\mu}_{x(S G)}$ is given by

$$
V\left(\hat{\mu}_{x(S G)}\right)=\frac{1}{n}\left[\sigma_{x}^{2}+w\left(\frac{\gamma_{s}^{2}}{\mu_{s}^{2}}\right)\left(\sigma_{x}^{2}+\mu_{x}^{2}\right)\right]
$$

PROOF. We have

$$
\begin{aligned}
V\left(\hat{\mu}_{x(S G)}\right) & =V\left(\frac{1}{n} \sum_{i=1}^{n} Z_{i}\right)^{2} \\
& =\frac{V\left(Z_{i}\right)}{n}
\end{aligned}
$$


Now,

$$
\begin{aligned}
V\left(Z_{i}\right) & =E\left(Z_{i}^{2}\right)-\left(E\left(Z_{i}\right)\right)^{2} \\
& =E\left(Z_{i}^{2}\right)-\mu_{x}^{2}
\end{aligned}
$$

Here

$$
\begin{aligned}
E\left(Z_{i}^{2}\right) & =E\left[\left\{\left(\frac{S}{\mu_{s}}\right)^{Y} X_{i}\right\}^{2} \mid Y=1\right] P(Y=1) \\
& +E\left[\left\{\left(\frac{S}{\mu_{s}}\right)^{Y} X_{i}\right\}^{2} \mid Y=0\right] P(Y=0) \\
& =E\left(\frac{S^{2} X_{i}^{2}}{\mu_{s}^{2}}\right) P(Y=1)+E\left(X_{i}^{2}\right) P(Y=0) \\
& =\frac{E\left(S^{2}\right) E\left(X_{i}^{2}\right)}{\mu_{s}^{2}} w+E\left(X_{i}^{2}\right)(1-w) \\
& =\frac{\left(\gamma_{s}^{2}+\mu_{s}^{2}\right)\left(\sigma_{x}^{2}+\mu_{x}^{2}\right)}{\mu_{s}^{2}} w+\left(\sigma_{x}^{2}+\mu_{x}^{2}\right)(1-w) \\
& =\left(1+\frac{\gamma_{s}^{2}}{\mu_{s}^{2}}\right)\left(\sigma_{x}^{2}+\mu_{x}^{2}\right) w+\left(\sigma_{x}^{2}+\mu_{x}^{2}\right)(1-w) \\
& =\left(\frac{\gamma_{s}^{2}}{\mu_{s}^{2}}\right)\left(\sigma_{x}^{2}+\mu_{x}^{2}\right) .
\end{aligned}
$$

From (2.5) and (2.6) we have

$$
\sigma_{z}^{2}=V\left(Z_{i}\right)=\sigma_{x}^{2}+w\left(\frac{\gamma_{s}^{2}}{\mu_{s}^{2}}\right)\left(\sigma_{x}^{2}+\mu_{x}^{2}\right)
$$


Thus from (2.4) and (2.7) we get

$$
V\left(\hat{\mu}_{x(S G)}\right)=\frac{1}{n}\left[\sigma_{x}^{2}+w\left(\frac{\gamma_{s}^{2}}{\mu_{s}^{2}}\right)\left(\sigma_{x}^{2}+\mu_{x}^{2}\right)\right]
$$

which proves the theorem.

The variance $\sigma_{x}^{2}$ of the sensitive variable $\mathrm{x}$ under the proposed randomization response procedure is obtained as follows:

$$
\begin{aligned}
& V\left(\hat{\mu}_{x(S G)}\right)=\frac{\sigma_{z}^{2}}{n}=(1 / n)\left[\sigma_{x}^{2}+w\left(\gamma_{s}^{2} / \mu_{s}^{2}\right)\left(\sigma_{x}^{2}+\mu_{x}^{2}\right)\right] \\
\Rightarrow & \sigma_{z}^{2}=\sigma_{x}^{2}\left(1+w C_{\gamma}^{2}\right)+w C_{\gamma}^{2} \mu_{x}^{2} \\
\Rightarrow & \sigma_{x}^{2}=\frac{\left(\sigma_{z}^{2}-w C_{\gamma}^{2} \mu_{x}^{2}\right)}{\left(1+w C_{\gamma}^{2}\right)},
\end{aligned}
$$

where $\sigma_{z}^{2}$ is defined in (2.7).

An estimator for $V\left(\hat{\mu}_{x(S G)}\right)$ is given by

$$
\hat{V}\left(\hat{\mu}_{x(S G)}\right)=(1 / n)\left[s_{x}^{2}+\hat{w}\left(\gamma_{s}^{2} / \mu_{s}^{2}\right)\left(s_{x}^{2}+\hat{\mu}_{x(S G)}^{2}\right)\right]
$$

where

$$
s_{x}^{2}=\frac{\left(s_{z}^{2}-\hat{w} C_{\gamma}^{2} \hat{\mu}_{x(S G)}^{2}\right)}{\left(1+\hat{w} C_{\gamma}^{2}\right)}
$$

with $s_{x}^{2}=\frac{1}{n-1} \sum_{i=1}^{n}\left(Z_{i}-\bar{Z}\right)^{2}$ is an estimator of $\sigma_{z}^{2}$.

The next section has been devoted to estimating $w$ based on the information gathered through proposed randomized response procedure. 


\section{ESTIMATION OF $w$}

Taking logarithm on both sides of (2.1) we have

$$
\log (Z)=Y \log (S / \theta)+\log (X)
$$

Taking expectation of both sides of (3.1) we have

$$
E[\log (Z)]=E(Y) E[\log (s)-\log (\theta)]+E[\log (X)]
$$

Replacing $X$ by $\hat{\mu}_{x(S G)}$ in (3.2), we get

$$
\begin{aligned}
E[\log (Z)] & \cong w E[\log (S)-\log (\theta)]+E\left[\log \left(\hat{\mu}_{x(S G)}\right)\right] \\
& =w E[\log (S)-\log (\theta)]+E[\log (\bar{Z})]
\end{aligned}
$$

Estimating $E(\log Z)$ by $\frac{1}{n} \sum_{i=1}^{n} \log Z_{i}$ and $E[\log (\bar{Z})]$ by $\frac{1}{n} \sum_{i=1}^{n} \log (\bar{Z})=\log (\bar{Z})$ in (3.3) leads to the estimator of $w$ given by

$$
\hat{w}=\frac{\frac{1}{n} \sum_{i=1}^{n} \log \left(Z_{i}\right)-\log \left(\frac{1}{n} \sum_{i=1}^{n} Z_{i}\right)}{\left(\delta-\mu_{s}\right)}, \delta \neq \mu_{s}
$$

where $\delta=E[\log (S)]$ denotes the known expected value of the logarithm of the scrambling variable $S$.

An estimator of the variance of $\hat{w}$ is given by

$$
\hat{V}(\hat{w})=\frac{\hat{w}(1-\hat{w})}{(n-1)}
$$

It follows by noting that $\hat{w}=n^{-1} \sum_{i=1}^{n} Y_{i}$ and $\sum_{i=1}^{n} Y_{i} \sim \operatorname{Binomial}(n, w)$. 


\section{EFFICIENCY COMPARISON}

From (1.2) and (1.5) we have

$$
V\left(\hat{\mu}_{x(E H)}\right)-V\left(\hat{\mu}_{x(G)}\right)=(1 / n) \gamma_{s}^{2}\left(\sigma_{x}^{2}+\mu_{x}^{2}\right)(1-w)
$$

which is always positive as $0 \leq w \leq 1$.

Thus we have the inequality:

$$
V\left(\hat{\mu}_{x(G)}\right)<V\left(\hat{\mu}_{x(E H)}\right)
$$

which shows that Gupta et al's (2002) estimator $\hat{\mu}_{x(G)}$ is more efficient than the Eichhorn and Hayre's (1983) estimator $\hat{\mu}_{x(E H)}$.

From (1.2) and (2.3) we have

$$
V\left(\hat{\mu}_{x(E H)}\right)-V\left(\hat{\mu}_{x(S G)}\right)=(1 / n) C_{\gamma}^{2}\left(\sigma_{x}^{2}+\mu_{x}^{2}\right)(1-w)
$$

which is always positive as $0 \leq w \leq 1$

Thus we have the inequality:

$$
V\left(\hat{\mu}_{x(S G)}\right)<V\left(\hat{\mu}_{x(E H)}\right)
$$

which follows that the proposed estimator $\hat{\mu}_{x(S G)}$ is more efficient than the Eichhorn and Hayre's (1983) estimator $\hat{\mu}_{x(E H)}$.

Further, from (1.5) and (2.3) we have

$$
V\left(\hat{\mu}_{x(G)}\right)-V\left(\hat{\mu}_{x(S G)}\right)=\frac{w\left(\sigma_{x}^{2}+\mu_{x}^{2}\right) \gamma_{s}^{2}}{n}\left(1-\frac{1}{\mu_{s}^{2}}\right)
$$


which is positive if

$$
1-\frac{1}{\mu_{s}^{2}}>0
$$

i.e. if $\mu_{s}^{2}>1$

i.e. if $\mu_{s}>1$

Thus we have the inequality:

$$
V\left(\hat{\mu}_{x(S G)}\right)<V\left(\hat{\mu}_{x(G)}\right), \mu_{s}>1
$$

It follows that the suggested randomization response procedure is always superior to Gupta et al.'s (2002) randomized response procedure as long as the condition $\mu_{s}>1$ (i.e. the mean $\mu_{s}$ of the scrambling variable $S$ is larger than the 'unity').

Further, from (4.4) and (4.7), we have the inequality:

$$
V\left(\hat{\mu}_{x(S G)}\right)<V\left(\hat{\mu}_{x(G)}\right)<V\left(\hat{\mu}_{x(E H)}\right), \mu_{s}>1
$$

It follows that when the mean $\mu_{s}$ of the scrambling variable $S$ is greater than the 'unity' (i.e. $\left.\mu_{s}>1\right)$ the proposed estimator $\hat{\mu}_{x(S G)}$ would be always better than the Eichhorn and Hayre (1983) estimator $\hat{\mu}_{x(E H)}$ and Gupta et al.'s (2002) estimator $\hat{\mu}_{x(G)}$. However, we note that the proposed randomized response model can be used in practice without imposing condition over the mean $\mu_{s}$ of the scrambling variables $S$. 


\section{EMPIRICAL STUDY}

To see the performance of the suggested estimator $\hat{\mu}_{x(S G)}$ over Eichhorn and Hayre's estimator $\hat{\mu}_{x(E H)}$ and Gupta et al.'s (2002) estimator $\hat{\mu}_{x(G)}$, we have computed the percent relative efficiency (PRE) of $\hat{\mu}_{x(S G)}$ with respect to $\hat{\mu}_{x(E H)}$ and $\hat{\mu}_{x(G)}$ respectively by using the following formulae:

$$
\begin{aligned}
& \operatorname{PRE}\left(\hat{\mu}_{x(S G)}, \hat{\mu}_{x(E H)}\right)=\frac{\left[C_{\gamma}^{2}+\left(\gamma_{s}^{2} / \mu_{s}^{2}\right)\left(1+C_{\gamma}^{2}\right)\right]}{\left[C_{\gamma}^{2}+w\left(\gamma_{s}^{2} / \mu_{s}^{2}\right)\left(1+C_{\gamma}^{2}\right)\right]} \times 100 \\
& \operatorname{PRE}\left(\hat{\mu}_{x(S G)}, \hat{\mu}_{x(G)}\right)=\frac{\left[C_{\gamma}^{2}+w \gamma_{s}^{2}\left(1+C_{\gamma}^{2}\right)\right]}{\left[C_{\gamma}^{2}+w\left(\gamma_{s}^{2} / \mu_{s}^{2}\right)\left(1+C_{\gamma}^{2}\right)\right]} \times 100
\end{aligned}
$$

for $w=0.2(0.2) 0.8, C_{\gamma}=0.1(0.2) 1.5, \gamma_{s}^{2}=5,10,15,20$ and $\mu_{s}=0.5(0.5) 3$. Findings are shown in Table 5.1 
Table 5.1 Value of $\operatorname{PRE}\left(\hat{\mu}_{x(S G)}, \hat{\mu}_{x(E H)}\right)$ and $\operatorname{PRE}\left(\hat{\mu}_{x(S G)}, \hat{\mu}_{x(G)}\right)$

\begin{tabular}{|c|c|c|c|c|}
\hline \multicolumn{5}{|c|}{$C_{\gamma}=0.1$} \\
\hline$\gamma_{s}^{2}$ & $\mu_{s}$ & $w$ & $\operatorname{PRE}\left(\hat{\mu}_{x(S G)}, \hat{\mu}_{x(E H)}\right)$ & $\operatorname{PRE}\left(\hat{\mu}_{x(S G)}, \hat{\mu}_{x(G)}\right)$ \\
\hline \multirow{4}{*}{5} & \multirow{4}{*}{0.5} & 0.2 & 995.57 & 25.37 \\
\hline & & 0.4 & 249.81 & 25.09 \\
\hline & & 0.6 & 166.61 & 25.06 \\
\hline & & 0.8 & 124.98 & 25.05 \\
\hline \multirow{4}{*}{5} & \multirow{4}{*}{1} & 0.2 & 496.08 & 100.00 \\
\hline & & 0.4 & 249.26 & 100.00 \\
\hline & & 0.6 & 166.45 & 100.00 \\
\hline & & 0.8 & 124.94 & 100.00 \\
\hline \multirow{4}{*}{5} & \multirow{4}{*}{1.5} & 0.2 & 491.28 & 222.28 \\
\hline & & 0.4 & 248.35 & 223.62 \\
\hline & & 0.6 & 166.18 & 224.08 \\
\hline & & 0.8 & 124.86 & 224.31 \\
\hline \multirow{4}{*}{5} & \multirow{4}{*}{2} & 0.2 & 484.76 & 388.57 \\
\hline & & 0.4 & 247.09 & 394.17 \\
\hline & & 0.6 & 165.80 & 396.09 \\
\hline & & 0.8 & 124.75 & 397.06 \\
\hline \multirow{4}{*}{5} & \multirow{4}{*}{2.5} & 0.2 & 476.69 & 594.41 \\
\hline & & 0.4 & 245.50 & 609.24 \\
\hline & & 0.6 & 165.32 & 614.39 \\
\hline & & 0.8 & 124.62 & 617.00 \\
\hline \multirow{4}{*}{5} & \multirow{4}{*}{3} & 0.2 & 467.27 & 834.55 \\
\hline & & 0.4 & 243.60 & 865.88 \\
\hline & & 0.6 & 164.74 & 876.92 \\
\hline & & 0.8 & 124.46 & 882.57 \\
\hline \multirow{4}{*}{10} & \multirow{4}{*}{0.5} & 0.2 & 499.51 & 25.09 \\
\hline & & 0.4 & 249.91 & 25.05 \\
\hline & & 0.6 & 166.64 & 25.03 \\
\hline & & 0.8 & 124.99 & 25.02 \\
\hline \multirow{4}{*}{10} & \multirow{4}{*}{1} & 0.2 & 498.03 & 100.00 \\
\hline & & 0.4 & 249.63 & 100.00 \\
\hline & & 0.6 & 166.56 & 100.00 \\
\hline & & 0.8 & 124.97 & 100.00 \\
\hline \multirow{4}{*}{10} & \multirow{4}{*}{1.5} & 0.2 & 495.59 & 223.62 \\
\hline & & 0.4 & 249.17 & 224.31 \\
\hline & & 0.6 & 166.42 & 224.54 \\
\hline & & 0.8 & 124.93 & 224.65 \\
\hline \multirow{4}{*}{10} & \multirow{4}{*}{2} & 0.2 & 492.23 & 394.17 \\
\hline & & 0.4 & 248.53 & 397.06 \\
\hline & & 0.6 & 166.23 & 398.03 \\
\hline & & 0.8 & 124.88 & 398.52 \\
\hline & & 0.2 & 488.00 & 609.24 \\
\hline 10 & 25 & 0.4 & 247.71 & 617.00 \\
\hline 10 & 2.3 & 0.6 & 165.99 & 619.64 \\
\hline & & 0.8 & 124.81 & 620.97 \\
\hline & & 0.2 & 482.94 & 865.88 \\
\hline 10 & 3 & 0.4 & 246.73 & 882.57 \\
\hline & & 0.6 & 165.69 & 888.29 \\
\hline & & 0.8 & 124.72 & 891.19 \\
\hline 15 & 05 & 0.2 & 499.67 & 25.06 \\
\hline 15 & 0.5 & 0.4 & 249.94 & 25.03 \\
\hline
\end{tabular}




\begin{tabular}{|c|c|c|c|c|}
\hline \multicolumn{5}{|c|}{$C_{\gamma}=0.1$} \\
\hline$\gamma_{s}^{2}$ & $\mu_{s}$ & $w$ & $\operatorname{PRE}\left(\hat{\mu}_{x(S G)}, \hat{\mu}_{x(E H)}\right)$ & $\operatorname{PRE}\left(\hat{\mu}_{x(S G)}, \hat{\mu}_{x(G)}\right)$ \\
\hline & & 0.6 & 166.65 & 25.02 \\
\hline & & 0.8 & 124.99 & 25.02 \\
\hline \multirow{4}{*}{15} & \multirow{4}{*}{1} & 0.2 & 498.68 & 100.00 \\
\hline & & 0.4 & 249.75 & 100.00 \\
\hline & & 0.6 & 166.59 & 100.00 \\
\hline & & 0.8 & 124.98 & 100.00 \\
\hline \multirow{4}{*}{15} & \multirow{4}{*}{1.5} & 0.2 & 497.05 & 224.08 \\
\hline & & 0.4 & 249.45 & 224.54 \\
\hline & & 0.6 & 166.50 & 224.69 \\
\hline & & 0.8 & 124.95 & 224.77 \\
\hline \multirow{4}{*}{15} & \multirow{4}{*}{2} & 0.2 & 494.79 & 396.09 \\
\hline & & 0.4 & 249.02 & 398.03 \\
\hline & & 0.6 & 166.37 & 398.69 \\
\hline & & 0.8 & 124.92 & 399.01 \\
\hline \multirow{4}{*}{15} & \multirow{4}{*}{2.5} & 0.2 & 491.92 & 614.39 \\
\hline & & 0.4 & 248.47 & 619.64 \\
\hline & & 0.6 & 166.21 & 621.41 \\
\hline & & 0.8 & 124.87 & 622.31 \\
\hline \multirow{4}{*}{15} & \multirow{4}{*}{3} & 0.2 & 488.46 & 876.92 \\
\hline & & 0.4 & 247.80 & 888.29 \\
\hline & & 0.6 & 166.01 & 892.16 \\
\hline & & 0.8 & 124.82 & 894.10 \\
\hline \multirow{4}{*}{20} & \multirow{4}{*}{0.5} & 0.2 & 499.75 & 25.05 \\
\hline & & 0.4 & 249.95 & 25.02 \\
\hline & & 0.6 & 166.65 & 25.02 \\
\hline & & 0.8 & 125.00 & 25.01 \\
\hline \multirow{4}{*}{20} & \multirow{4}{*}{1} & 0.2 & 499.01 & 100.00 \\
\hline & & 0.4 & 249.81 & 100.00 \\
\hline & & 0.6 & 166.61 & 100.00 \\
\hline & & 0.8 & 124.98 & 100.00 \\
\hline \multirow{4}{*}{20} & \multirow{4}{*}{1.5} & 0.2 & 497.78 & 224.31 \\
\hline & & 0.4 & 249.58 & 224.65 \\
\hline & & 0.6 & 166.54 & 224.77 \\
\hline & & 0.8 & 124.97 & 224.83 \\
\hline \multirow{4}{*}{20} & \multirow{4}{*}{2} & 0.2 & 496.08 & 397.06 \\
\hline & & 0.4 & 249.26 & 398.52 \\
\hline & & 0.6 & 166.45 & 399.01 \\
\hline & & 0.8 & 124.94 & 399.26 \\
\hline \multirow{4}{*}{20} & \multirow{4}{*}{2.5} & 0.2 & 493.91 & 617.00 \\
\hline & & 0.4 & 248.85 & 620.97 \\
\hline & & 0.6 & 166.32 & 622.31 \\
\hline & & 0.8 & 124.90 & 622.98 \\
\hline \multirow{4}{*}{20} & \multirow{4}{*}{3} & 0.2 & 491.28 & 882.57 \\
\hline & & 0.4 & 248.35 & 891.19 \\
\hline & & 0.6 & 166.18 & 894.10 \\
\hline & & 0.8 & 124.86 & 895.57 \\
\hline
\end{tabular}

Table 5.1 Continued 


\begin{tabular}{|c|c|c|c|c|}
\hline \multicolumn{5}{|c|}{$C_{\gamma}=0.3$} \\
\hline$\gamma_{s}^{2}$ & $\mu_{s}$ & $w$ & $\operatorname{PRE}\left(\hat{\mu}_{x(S G)}, \hat{\mu}_{x(E H)}\right)$ & $\operatorname{PRE}\left(\hat{\mu}_{x(S G)}, \hat{\mu}_{x(G)}\right)$ \\
\hline \multirow{4}{*}{5} & \multirow{4}{*}{0.5} & 0.2 & 491.91 & 26.52 \\
\hline & & 0.4 & 248.47 & 25.77 \\
\hline & & 0.6 & 166.21 & 25.51 \\
\hline & & 0.8 & 124.87 & 25.39 \\
\hline \multirow{4}{*}{5} & \multirow{4}{*}{1} & 0.2 & 469.49 & 100.00 \\
\hline & & 0.4 & 244.05 & 100.00 \\
\hline & & 0.6 & 164.88 & 100.00 \\
\hline & & 0.8 & 124.49 & 100.00 \\
\hline \multirow{4}{*}{5} & \multirow{4}{*}{1.5} & 0.2 & 437.33 & 205.42 \\
\hline & & 0.4 & 237.25 & 214.38 \\
\hline & & 0.6 & 162.78 & 217.71 \\
\hline & & 0.8 & 123.89 & 219.45 \\
\hline \multirow{4}{*}{5} & \multirow{4}{*}{2} & 0.2 & 400.69 & 325.52 \\
\hline & & 0.4 & 228.74 & 357.48 \\
\hline & & 0.6 & 160.06 & 370.25 \\
\hline & & 0.8 & 123.09 & 377.12 \\
\hline \multirow{4}{*}{5} & \multirow{4}{*}{2.5} & 0.2 & 363.84 & 446.29 \\
\hline & & 0.4 & 219.23 & 517.32 \\
\hline & & 0.6 & 156.88 & 547.95 \\
\hline & & 0.8 & 122.14 & 565.01 \\
\hline \multirow{4}{*}{5} & \multirow{4}{*}{3} & 0.2 & 329.47 & 558.95 \\
\hline & & 0.4 & 209.36 & 683.28 \\
\hline & & 0.6 & 153.43 & 741.18 \\
\hline & & 0.8 & 121.08 & 774.66 \\
\hline \multirow{4}{*}{10} & \multirow{4}{*}{0.5} & 0.2 & 495.91 & 25.77 \\
\hline & & 0.4 & 249.23 & 25.39 \\
\hline & & 0.6 & 166.44 & 25.26 \\
\hline & & 0.8 & 124.94 & 25.19 \\
\hline \multirow{4}{*}{10} & \multirow{4}{*}{1} & 0.2 & 484.14 & 100.00 \\
\hline & & 0.4 & 246.97 & 100.00 \\
\hline & & 0.6 & 165.76 & 100.00 \\
\hline & & 0.8 & 124.74 & 100.00 \\
\hline \multirow{4}{*}{10} & \multirow{4}{*}{1.5} & 0.2 & 466.00 & 214.38 \\
\hline & & 0.4 & 243.34 & 219.45 \\
\hline & & 0.6 & 164.66 & 221.25 \\
\hline & & 0.8 & 124.43 & 222.16 \\
\hline \multirow{4}{*}{10} & & 0.2 & 443.31 & 357.48 \\
\hline & & 0.4 & 238.56 & 377.12 \\
\hline & 2 & 0.6 & 163.19 & 384.35 \\
\hline & & 0.8 & 124.01 & 388.11 \\
\hline & & 0.2 & 417.96 & 517.32 \\
\hline 11 & 25 & 0.4 & 232.86 & 565.01 \\
\hline 10 & 2.5 & 0.6 & 161.39 & 583.42 \\
\hline & & 0.8 & 123.49 & 593.19 \\
\hline & & 0.2 & 391.64 & 683.28 \\
\hline 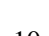 & 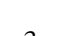 & 0.4 & 226.50 & 774.66 \\
\hline 10 & 3 & 0.6 & 159.32 & 811.84 \\
\hline & & 0.8 & 122.88 & 832.00 \\
\hline & & 0.2 & 497.27 & 25.51 \\
\hline 15 & 0.5 & 0.4 & 249.49 & 25.26 \\
\hline 15 & 0.5 & 0.6 & 166.51 & 25.17 \\
\hline & & 0.8 & 124.96 & 25.13 \\
\hline
\end{tabular}




\begin{tabular}{|c|c|c|c|c|}
\hline \multicolumn{5}{|c|}{$C_{\gamma}=0.3$} \\
\hline$\gamma_{s}^{2}$ & $\mu_{s}$ & $w$ & $\operatorname{PRE}\left(\hat{\mu}_{x(S G)}, \hat{\mu}_{x(E H)}\right)$ & $\operatorname{PRE}\left(\hat{\mu}_{x(S G)}, \hat{\mu}_{x(G)}\right)$ \\
\hline \multirow{4}{*}{15} & \multirow{4}{*}{1} & 0.2 & 489.29 & 100.00 \\
\hline & & 0.4 & 247.96 & 100.00 \\
\hline & & 0.6 & 166.06 & 100.00 \\
\hline & & 0.8 & 124.83 & 100.00 \\
\hline \multirow{4}{*}{15} & \multirow{4}{*}{1.5} & 0.2 & 476.67 & 217.71 \\
\hline & & 0.4 & 245.49 & 221.25 \\
\hline & & 0.6 & 165.32 & 222.47 \\
\hline & & 0.8 & 124.62 & 223.09 \\
\hline \multirow{4}{*}{15} & \multirow{4}{*}{2} & 0.2 & 460.33 & 370.25 \\
\hline & & 0.4 & 242.17 & 384.35 \\
\hline & & 0.6 & 164.31 & 389.38 \\
\hline & & 0.8 & 124.33 & 391.96 \\
\hline \multirow{4}{*}{15} & \multirow{4}{*}{2.5} & 0.2 & 441.29 & 547.95 \\
\hline & & 0.4 & 238.12 & 583.42 \\
\hline & & 0.6 & 163.05 & 596.53 \\
\hline & & 0.8 & 123.97 & 603.35 \\
\hline \multirow{4}{*}{15} & \multirow{4}{*}{3} & 0.2 & 420.59 & 741.18 \\
\hline & & 0.4 & 233.47 & 811.84 \\
\hline & & 0.6 & 161.58 & 838.98 \\
\hline & & 0.8 & 123.54 & 853.35 \\
\hline \multirow{4}{*}{20} & \multirow{4}{*}{0.5} & 0.2 & 497.95 & 25.39 \\
\hline & & 0.4 & 249.61 & 25.19 \\
\hline & & 0.6 & 166.55 & 25.13 \\
\hline & & 0.8 & 124.97 & 25.10 \\
\hline \multirow{4}{*}{20} & \multirow{4}{*}{1} & 0.2 & 491.91 & 100.00 \\
\hline & & 0.4 & 248.47 & 100.00 \\
\hline & & 0.6 & 166.21 & 100.00 \\
\hline & & 0.8 & 124.87 & 100.00 \\
\hline \multirow{4}{*}{20} & \multirow{4}{*}{1.5} & 0.2 & 482.25 & 219.45 \\
\hline & & 0.4 & 246.60 & 222.16 \\
\hline & & 0.6 & 165.65 & 223.09 \\
\hline & & 0.8 & 124.71 & 223.57 \\
\hline \multirow{4}{*}{20} & \multirow{4}{*}{2} & 0.2 & 469.49 & 377.12 \\
\hline & & 0.4 & 244.05 & 388.11 \\
\hline & & 0.6 & 164.88 & 391.96 \\
\hline & & 0.8 & 124.49 & 393.93 \\
\hline \multirow{4}{*}{20} & \multirow{4}{*}{2.5} & 0.2 & 454.29 & 565.01 \\
\hline & & 0.4 & 240.91 & 593.19 \\
\hline & & 0.6 & 163.92 & 603.35 \\
\hline & & 0.8 & 124.22 & 608.60 \\
\hline \multirow{4}{*}{20} & \multirow{4}{*}{3} & 0.2 & 437.33 & 774.66 \\
\hline & & 0.4 & 237.25 & 832.00 \\
\hline & & 0.6 & 162.78 & 853.35 \\
\hline & & 0.8 & 123.89 & 864.49 \\
\hline
\end{tabular}

Table 5.1 Continued 


\begin{tabular}{|c|c|c|c|c|}
\hline \multicolumn{5}{|c|}{$C_{\gamma}=0.5$} \\
\hline$\gamma_{s}^{2}$ & $\mu_{s}$ & $w$ & $\operatorname{PRE}\left(\hat{\mu}_{x(S G)}, \hat{\mu}_{x(E H)}\right)$ & $\operatorname{PRE}\left(\hat{\mu}_{x(S G)}, \hat{\mu}_{x(G)}\right)$ \\
\hline \multirow{4}{*}{5} & \multirow{4}{*}{0.5} & 0.2 & 480.95 & 28.57 \\
\hline & & 0.4 & 246.34 & 26.83 \\
\hline & & 0.6 & 165.57 & 26.23 \\
\hline & & 0.8 & 124.69 & 25.93 \\
\hline \multirow{4}{*}{5} & \multirow{4}{*}{1} & 0.2 & 433.33 & 100.00 \\
\hline & & 0.4 & 236.36 & 100.00 \\
\hline & & 0.6 & 162.50 & 100.00 \\
\hline & & 0.8 & 123.81 & 100.00 \\
\hline \multirow{4}{*}{5} & \multirow{4}{*}{1.5} & 0.2 & 375.86 & 186.21 \\
\hline & & 0.4 & 222.45 & 202.04 \\
\hline & & 0.6 & 157.97 & 208.70 \\
\hline & & 0.8 & 122.47 & 212.36 \\
\hline \multirow{4}{*}{5} & \multirow{4}{*}{2} & 0.2 & 322.22 & 266.67 \\
\hline & & 0.4 & 207.14 & 314.29 \\
\hline & & 0.6 & 152.63 & 336.84 \\
\hline & & 0.8 & 120.83 & 350.00 \\
\hline \multirow{4}{*}{5} & \multirow{4}{*}{2.5} & 0.2 & 277.78 & 333.33 \\
\hline & & 0.4 & 192.31 & 423.08 \\
\hline & & 0.6 & 147.06 & 470.59 \\
\hline & & 0.8 & 119.05 & 500.00 \\
\hline \multirow{4}{*}{5} & \multirow{4}{*}{3} & 0.2 & 242.86 & 385.71 \\
\hline & & 0.4 & 178.95 & 521.05 \\
\hline & & 0.6 & 141.67 & 600.00 \\
\hline & & 0.8 & 117.24 & 651.72 \\
\hline \multirow{4}{*}{10} & \multirow{4}{*}{0.5} & 0.2 & 490.24 & 26.83 \\
\hline & & 0.4 & 248.15 & 25.93 \\
\hline & & 0.6 & 166.12 & 25.62 \\
\hline & & 0.8 & 124.84 & 25.47 \\
\hline \multirow{4}{*}{10} & \multirow{4}{*}{1} & 0.2 & 463.64 & 100.00 \\
\hline & & 0.4 & 242.86 & 100.00 \\
\hline & & 0.6 & 164.52 & 100.00 \\
\hline & & 0.8 & 124.39 & 100.00 \\
\hline \multirow{4}{*}{10} & \multirow{4}{*}{1.5} & 0.2 & 426.53 & 202.04 \\
\hline & & 0.4 & 234.83 & 212.36 \\
\hline & & 0.6 & 162.02 & 216.28 \\
\hline & & 0.8 & 123.67 & 218.34 \\
\hline \multirow{4}{*}{10} & & 0.2 & 385.71 & 314.29 \\
\hline & 2 & 0.4 & 225.00 & 350.00 \\
\hline & 2 & 0.6 & 158.82 & 364.71 \\
\hline & & 0.8 & 122.73 & 372.73 \\
\hline & & 0.2 & 346.15 & 423.08 \\
\hline 10 & 25 & 0.4 & 214.29 & 500.00 \\
\hline 10 & 2.3 & 0.6 & 155.17 & 534.48 \\
\hline & & 0.8 & 121.62 & 554.05 \\
\hline & & 0.2 & 310.53 & 521.05 \\
\hline 10 & 3 & 0.4 & 203.45 & 651.72 \\
\hline 10 & 3 & 0.6 & 151.28 & 715.38 \\
\hline & & 0.8 & 120.41 & 753.06 \\
\hline & & 0.2 & 493.44 & 26.23 \\
\hline 15 & 05 & 0.4 & 248.76 & 25.62 \\
\hline 15 & 0.5 & 0.6 & 166.30 & 25.41 \\
\hline & & 0.8 & 124.90 & 25.31 \\
\hline
\end{tabular}




\begin{tabular}{|c|c|c|c|c|}
\hline \multicolumn{5}{|c|}{$C_{\gamma}=0.5$} \\
\hline$\gamma_{s}^{2}$ & $\mu_{s}$ & $w$ & $\operatorname{PRE}\left(\hat{\mu}_{x(S G)}, \hat{\mu}_{x(E H)}\right)$ & $\operatorname{PRE}\left(\hat{\mu}_{x(S G)}, \hat{\mu}_{x(G)}\right)$ \\
\hline \multirow{4}{*}{15} & \multirow{4}{*}{1} & 0.2 & 475.00 & 100.00 \\
\hline & & 0.4 & 245.16 & 100.00 \\
\hline & & 0.6 & 165.22 & 100.00 \\
\hline & & 0.8 & 124.59 & 100.00 \\
\hline \multirow{4}{*}{15} & \multirow{4}{*}{1.5} & 0.2 & 447.83 & 208.70 \\
\hline & & 0.4 & 239.53 & 216.28 \\
\hline & & 0.6 & 163.49 & 219.05 \\
\hline & & 0.8 & 124.10 & 220.48 \\
\hline \multirow{4}{*}{15} & \multirow{4}{*}{2} & 0.2 & 415.79 & 336.84 \\
\hline & & 0.4 & 232.35 & 364.71 \\
\hline & & 0.6 & 161.22 & 375.51 \\
\hline & & 0.8 & 123.44 & 381.25 \\
\hline \multirow{4}{*}{15} & \multirow{4}{*}{2.5} & 0.2 & 382.35 & 470.59 \\
\hline & & 0.4 & 224.14 & 534.48 \\
\hline & & 0.6 & 158.54 & 560.98 \\
\hline & & 0.8 & 122.64 & 575.47 \\
\hline \multirow{4}{*}{15} & \multirow{4}{*}{3} & 0.2 & 350.00 & 600.00 \\
\hline & & 0.4 & 215.38 & 715.38 \\
\hline & & 0.6 & 155.56 & 766.67 \\
\hline & & 0.8 & 121.74 & 795.65 \\
\hline \multirow{4}{*}{20} & \multirow{4}{*}{0.5} & 0.2 & 495.06 & 25.93 \\
\hline & & 0.4 & 249.07 & 25.47 \\
\hline & & 0.6 & 166.39 & 25.31 \\
\hline & & 0.8 & 124.92 & 25.23 \\
\hline \multirow{4}{*}{20} & \multirow{4}{*}{1} & 0.2 & 480.95 & 100.00 \\
\hline & & 0.4 & 246.34 & 100.00 \\
\hline & & 0.6 & 165.57 & 100.00 \\
\hline & & 0.8 & 124.69 & 100.00 \\
\hline \multirow{4}{*}{20} & \multirow{4}{*}{1.5} & 0.2 & 459.55 & 212.36 \\
\hline & & 0.4 & 242.01 & 218.34 \\
\hline & & 0.6 & 164.26 & 220.48 \\
\hline & & 0.8 & 124.32 & 221.58 \\
\hline \multirow{4}{*}{20} & \multirow{4}{*}{2} & 0.2 & 433.33 & 350.00 \\
\hline & & 0.4 & 236.36 & 372.73 \\
\hline & & 0.6 & 162.50 & 381.25 \\
\hline & & 0.8 & 123.81 & 385.71 \\
\hline \multirow{4}{*}{20} & \multirow{4}{*}{2.5} & 0.2 & 404.76 & 500.00 \\
\hline & & 0.4 & 229.73 & 554.05 \\
\hline & & 0.6 & 160.38 & 575.47 \\
\hline & & 0.8 & 123.19 & 586.96 \\
\hline \multirow{4}{*}{20} & \multirow{4}{*}{3} & 0.2 & 375.86 & 651.72 \\
\hline & & 0.4 & 222.45 & 753.06 \\
\hline & & 0.6 & 157.97 & 795.65 \\
\hline & & 0.8 & 122.47 & 819.10 \\
\hline
\end{tabular}

Table 5.1 Continued 


\begin{tabular}{|c|c|c|c|c|}
\hline \multicolumn{5}{|c|}{$C_{\gamma}=0.7$} \\
\hline$\gamma_{s}^{2}$ & $\mu_{s}$ & $w$ & $\operatorname{PRE}\left(\hat{\mu}_{x(S G)}, \hat{\mu}_{x(E H)}\right)$ & $\operatorname{PRE}\left(\hat{\mu}_{x(S G)}, \hat{\mu}_{x(G)}\right)$ \\
\hline \multirow{4}{*}{5} & \multirow{4}{*}{0.5} & 0.2 & 469.61 & 30.70 \\
\hline & & 0.4 & 244.08 & 27.96 \\
\hline & & 0.6 & 164.89 & 27.00 \\
\hline & & 0.8 & 124.50 & 26.51 \\
\hline \multirow{4}{*}{5} & \multirow{4}{*}{1} & 0.2 & 401.01 & 100.00 \\
\hline & & 0.4 & 228.82 & 100.00 \\
\hline & & 0.6 & 160.08 & 100.00 \\
\hline & & 0.8 & 123.10 & 100.00 \\
\hline \multirow{4}{*}{5} & \multirow{4}{*}{1.5} & 0.2 & 329.89 & 171.84 \\
\hline & & 0.4 & 209.49 & 191.24 \\
\hline & & 0.6 & 153.48 & 200.27 \\
\hline & & 0.8 & 121.10 & 205.49 \\
\hline \multirow{4}{*}{5} & \multirow{4}{*}{2} & 0.2 & 272.75 & 229.57 \\
\hline & & 0.4 & 190.49 & 280.97 \\
\hline & & 0.6 & 146.35 & 308.55 \\
\hline & & 0.8 & 118.81 & 325.76 \\
\hline \multirow{4}{*}{5} & \multirow{4}{*}{2.5} & 0.2 & 230.92 & 271.83 \\
\hline & & 0.4 & 173.98 & 358.92 \\
\hline & & 0.6 & 139.56 & 411.55 \\
\hline & & 0.8 & 116.51 & 446.80 \\
\hline \multirow{4}{*}{5} & \multirow{4}{*}{3} & 0.2 & 201.02 & 302.03 \\
\hline & & 0.4 & 160.49 & 422.60 \\
\hline & & 0.6 & 133.56 & 502.70 \\
\hline & & 0.8 & 114.37 & 559.79 \\
\hline \multirow{4}{*}{10} & \multirow{4}{*}{0.5} & 0.2 & 484.21 & 27.96 \\
\hline & & 0.4 & 246.98 & 26.51 \\
\hline & & 0.6 & 165.77 & 26.01 \\
\hline & & 0.8 & 124.75 & 25.76 \\
\hline \multirow{4}{*}{10} & \multirow{4}{*}{1} & 0.2 & 443.52 & 100.00 \\
\hline & & 0.4 & 238.60 & 100.00 \\
\hline & & 0.6 & 163.20 & 100.00 \\
\hline & & 0.8 & 124.01 & 100.00 \\
\hline \multirow{4}{*}{10} & \multirow{4}{*}{1.5} & 0.2 & 391.98 & 191.24 \\
\hline & & 0.4 & 226.58 & 205.49 \\
\hline & & 0.6 & 159.35 & 211.28 \\
\hline & & 0.8 & 122.88 & 214.42 \\
\hline \multirow{4}{*}{10} & & 0.2 & 341.30 & 280.97 \\
\hline & 2 & 0.4 & 212.88 & 325.76 \\
\hline & 2 & 0.6 & 154.68 & 346.06 \\
\hline & & 0.8 & 121.47 & 357.64 \\
\hline & & 0.2 & 297.27 & 358.92 \\
\hline & & 0.4 & 199.09 & 446.80 \\
\hline 10 & 2.5 & 0.6 & 149.66 & 491.04 \\
\hline & & 0.8 & 119.89 & 517.69 \\
\hline & & 0.2 & 261.30 & 422.60 \\
\hline 10 & 3 & 0.4 & 186.21 & 559.79 \\
\hline 10 & 3 & 0.6 & 144.64 & 635.73 \\
\hline & & 0.8 & 118.25 & 683.96 \\
\hline & & 0.2 & 489.33 & 27.00 \\
\hline 15 & 05 & 0.4 & 247.97 & 26.01 \\
\hline 15 & 0.5 & 0.6 & 166.06 & 25.68 \\
\hline & & 0.8 & 124.83 & 25.51 \\
\hline
\end{tabular}




\begin{tabular}{|c|c|c|c|c|}
\hline \multicolumn{5}{|c|}{$C_{\gamma}=0.7$} \\
\hline$\gamma_{s}^{2}$ & $\mu_{s}$ & $w$ & $\operatorname{PRE}\left(\hat{\mu}_{x(S G)}, \hat{\mu}_{x(E H)}\right)$ & $\operatorname{PRE}\left(\hat{\mu}_{x(S G)}, \hat{\mu}_{x(G)}\right)$ \\
\hline \multirow{4}{*}{15} & \multirow{4}{*}{1} & 0.2 & 460.48 & 100.00 \\
\hline & & 0.4 & 242.21 & 100.00 \\
\hline & & 0.6 & 164.32 & 100.00 \\
\hline & & 0.8 & 124.33 & 100.00 \\
\hline \multirow{4}{*}{15} & \multirow{4}{*}{1.5} & 0.2 & 420.86 & 200.27 \\
\hline & & 0.4 & 233.53 & 211.28 \\
\hline & & 0.6 & 161.60 & 215.50 \\
\hline & & 0.8 & 123.55 & 217.74 \\
\hline \multirow{4}{*}{15} & \multirow{4}{*}{2} & 0.2 & 378.07 & 308.55 \\
\hline & & 0.4 & 223.03 & 346.06 \\
\hline & & 0.6 & 158.17 & 361.74 \\
\hline & & 0.8 & 122.53 & 370.36 \\
\hline \multirow{4}{*}{15} & \multirow{4}{*}{2.5} & 0.2 & 337.37 & 411.55 \\
\hline & & 0.4 & 211.73 & 491.04 \\
\hline & & 0.6 & 154.27 & 527.39 \\
\hline & & 0.8 & 121.34 & 548.23 \\
\hline \multirow{4}{*}{15} & \multirow{4}{*}{3} & 0.2 & 301.35 & 502.70 \\
\hline & & 0.4 & 200.45 & 635.73 \\
\hline & & 0.6 & 150.17 & 702.02 \\
\hline & & 0.8 & 120.05 & 741.72 \\
\hline \multirow{4}{*}{20} & \multirow{4}{*}{0.5} & 0.2 & 491.94 & 26.51 \\
\hline & & 0.4 & 248.47 & 25.76 \\
\hline & & 0.6 & 166.21 & 25.51 \\
\hline & & 0.8 & 124.87 & 25.38 \\
\hline \multirow{4}{*}{20} & \multirow{4}{*}{1} & 0.2 & 469.61 & 100.00 \\
\hline & & 0.4 & 244.08 & 100.00 \\
\hline & & 0.6 & 164.89 & 100.00 \\
\hline & & 0.8 & 124.50 & 100.00 \\
\hline \multirow{4}{*}{20} & \multirow{4}{*}{1.5} & 0.2 & 437.56 & 205.49 \\
\hline & & 0.4 & 237.30 & 214.42 \\
\hline & & 0.6 & 162.79 & 217.74 \\
\hline & & 0.8 & 123.89 & 219.47 \\
\hline \multirow{4}{*}{20} & \multirow{4}{*}{2} & 0.2 & 401.01 & 325.76 \\
\hline & & 0.4 & 228.82 & 357.64 \\
\hline & & 0.6 & 160.08 & 370.36 \\
\hline & & 0.8 & 123.10 & 377.21 \\
\hline \multirow{4}{*}{20} & \multirow{4}{*}{2.5} & 0.2 & 364.23 & 446.80 \\
\hline & & 0.4 & 219.34 & 517.69 \\
\hline & & 0.6 & 156.92 & 548.23 \\
\hline & & 0.8 & 122.15 & 565.24 \\
\hline \multirow{4}{*}{20} & \multirow{4}{*}{3} & 0.2 & 329.89 & 559.79 \\
\hline & & 0.4 & 209.49 & 683.96 \\
\hline & & 0.6 & 153.48 & 741.72 \\
\hline & & 0.8 & 121.10 & 775.12 \\
\hline
\end{tabular}




\begin{tabular}{|c|c|c|c|c|}
\hline \multicolumn{5}{|c|}{$C_{\gamma}=0.9$} \\
\hline$\gamma_{s}^{2}$ & $\mu_{s}$ & $w$ & $\operatorname{PRE}\left(\hat{\mu}_{x(S G)}, \hat{\mu}_{x(E H)}\right)$ & $\operatorname{PRE}\left(\hat{\mu}_{x(S G)}, \hat{\mu}_{x(G)}\right)$ \\
\hline \multirow{4}{*}{5} & \multirow{4}{*}{0.5} & 0.2 & 459.75 & 32.55 \\
\hline & & 0.4 & 242.05 & 28.97 \\
\hline & & 0.6 & 164.27 & 27.70 \\
\hline & & 0.8 & 124.32 & 27.04 \\
\hline \multirow{4}{*}{5} & \multirow{4}{*}{1} & 0.2 & 376.34 & 100.00 \\
\hline & & 0.4 & 222.57 & 100.00 \\
\hline & & 0.6 & 158.01 & 100.00 \\
\hline & & 0.8 & 122.48 & 100.00 \\
\hline \multirow{4}{*}{5} & \multirow{4}{*}{1.5} & 0.2 & 299.31 & 162.28 \\
\hline & & 0.4 & 199.77 & 183.14 \\
\hline & & 0.6 & 149.91 & 193.59 \\
\hline & & 0.8 & 119.97 & 199.86 \\
\hline \multirow{4}{*}{5} & \multirow{4}{*}{2} & 0.2 & 243.37 & 207.52 \\
\hline & & 0.4 & 179.15 & 258.31 \\
\hline & & 0.6 & 141.75 & 287.89 \\
\hline & & 0.8 & 117.27 & 307.25 \\
\hline \multirow{4}{*}{5} & \multirow{4}{*}{2.5} & 0.2 & 205.35 & 238.27 \\
\hline & & 0.4 & 162.54 & 318.89 \\
\hline & & 0.6 & 134.50 & 371.69 \\
\hline & & 0.8 & 114.71 & 408.96 \\
\hline \multirow{4}{*}{5} & \multirow{4}{*}{3} & 0.2 & 179.56 & 259.12 \\
\hline & & 0.4 & 149.77 & 365.44 \\
\hline & & 0.6 & 128.46 & 441.51 \\
\hline & & 0.8 & 112.46 & 498.62 \\
\hline \multirow{4}{*}{10} & \multirow{4}{*}{0.5} & 0.2 & 478.81 & 28.97 \\
\hline & & 0.4 & 245.92 & 27.04 \\
\hline & & 0.6 & 165.45 & 26.37 \\
\hline & & 0.8 & 124.66 & 26.03 \\
\hline \multirow{4}{*}{10} & \multirow{4}{*}{1} & 0.2 & 426.86 & 100.00 \\
\hline & & 0.4 & 234.91 & 100.00 \\
\hline & & 0.6 & 162.04 & 100.00 \\
\hline & & 0.8 & 123.68 & 100.00 \\
\hline \multirow{4}{*}{10} & \multirow{4}{*}{1.5} & 0.2 & 366.05 & 183.14 \\
\hline & & 0.4 & 219.83 & 199.86 \\
\hline & & 0.6 & 157.09 & 207.04 \\
\hline & & 0.8 & 122.21 & 211.03 \\
\hline \multirow{4}{*}{10} & & 0.2 & 311.08 & 258.31 \\
\hline & 2 & 0.4 & 203.63 & 307.25 \\
\hline & 2 & 0.6 & 151.35 & 331.06 \\
\hline & & 0.8 & 120.43 & 345.15 \\
\hline & & 0.2 & 266.77 & 318.89 \\
\hline 10 & 25 & 0.4 & 188.27 & 408.96 \\
\hline 10 & 2.5 & 0.6 & 145.47 & 458.08 \\
\hline & & 0.8 & 118.52 & 489.00 \\
\hline & & 0.2 & 232.72 & 365.44 \\
\hline 10 & 2 & 0.4 & 174.74 & 498.62 \\
\hline 10 & 3 & 0.6 & 139.89 & 578.68 \\
\hline & & 0.8 & 116.63 & 632.11 \\
\hline & & 0.2 & 485.62 & 27.70 \\
\hline 15 & 05 & 0.4 & 247.25 & 26.37 \\
\hline 15 & 0.5 & 0.6 & 165.85 & 25.92 \\
\hline & & 0.8 & 124.77 & 25.69 \\
\hline
\end{tabular}




\begin{tabular}{|c|c|c|c|c|}
\hline \multicolumn{5}{|c|}{$C_{\gamma}=0.9$} \\
\hline$\gamma_{s}^{2}$ & $\mu_{s}$ & $w$ & $\operatorname{PRE}\left(\hat{\mu}_{x(S G)}, \hat{\mu}_{x(E H)}\right)$ & $\operatorname{PRE}\left(\hat{\mu}_{x(S G)}, \hat{\mu}_{x(G)}\right)$ \\
\hline \multirow{4}{*}{15} & \multirow{4}{*}{1} & 0.2 & 448.08 & 100.00 \\
\hline & & 0.4 & 239.59 & 100.00 \\
\hline & & 0.6 & 163.51 & 100.00 \\
\hline & & 0.8 & 124.10 & 100.00 \\
\hline \multirow{4}{*}{15} & \multirow{4}{*}{1.5} & 0.2 & 399.48 & 193.59 \\
\hline & & 0.4 & 228.44 & 207.04 \\
\hline & & 0.6 & 159.96 & 212.42 \\
\hline & & 0.8 & 123.06 & 215.32 \\
\hline \multirow{4}{*}{15} & \multirow{4}{*}{2} & 0.2 & 350.52 & 287.89 \\
\hline & & 0.4 & 215.53 & 331.06 \\
\hline & & 0.6 & 155.61 & 350.23 \\
\hline & & 0.8 & 121.75 & 361.06 \\
\hline \multirow{4}{*}{15} & \multirow{4}{*}{2.5} & 0.2 & 307.01 & 371.69 \\
\hline & & 0.4 & 202.31 & 458.08 \\
\hline & & 0.6 & 150.86 & 500.53 \\
\hline & & 0.8 & 120.27 & 525.76 \\
\hline \multirow{4}{*}{15} & \multirow{4}{*}{3} & 0.2 & 270.75 & 441.51 \\
\hline & & 0.4 & 189.75 & 578.68 \\
\hline & & 0.6 & 146.06 & 652.67 \\
\hline & & 0.8 & 118.72 & 698.97 \\
\hline \multirow{4}{*}{20} & \multirow{4}{*}{0.5} & 0.2 & 489.12 & 27.04 \\
\hline & & 0.4 & 247.93 & 26.03 \\
\hline & & 0.6 & 166.05 & 25.69 \\
\hline & & 0.8 & 124.83 & 25.52 \\
\hline \multirow{4}{*}{20} & \multirow{4}{*}{1} & 0.2 & 459.75 & 100.00 \\
\hline & & 0.4 & 242.05 & 100.00 \\
\hline & & 0.6 & 164.27 & 100.00 \\
\hline & & 0.8 & 124.32 & 100.00 \\
\hline \multirow{4}{*}{20} & \multirow{4}{*}{1.5} & 0.2 & 419.56 & 199.86 \\
\hline & & 0.4 & 233.23 & 211.03 \\
\hline & & 0.6 & 161.51 & 215.32 \\
\hline & & 0.8 & 123.52 & 217.60 \\
\hline \multirow{4}{*}{20} & \multirow{4}{*}{2} & 0.2 & 376.34 & 307.25 \\
\hline & & 0.4 & 222.57 & 345.15 \\
\hline & & 0.6 & 158.01 & 361.06 \\
\hline & & 0.8 & 122.48 & 369.81 \\
\hline \multirow{4}{*}{20} & \multirow{4}{*}{2.5} & 0.2 & 335.40 & 408.96 \\
\hline & & 0.4 & 211.14 & 489.00 \\
\hline & & 0.6 & 154.07 & 525.76 \\
\hline & & 0.8 & 121.28 & 546.88 \\
\hline \multirow{4}{*}{20} & \multirow{4}{*}{3} & 0.2 & 299.31 & 498.62 \\
\hline & & 0.4 & 199.77 & 632.11 \\
\hline & & 0.6 & 149.91 & 698.97 \\
\hline & & 0.8 & 119.97 & 739.12 \\
\hline
\end{tabular}

Table 5.1 Continued 


\begin{tabular}{|c|c|c|c|c|}
\hline \multicolumn{5}{|c|}{$C_{\gamma}=1.1$} \\
\hline$\gamma_{s}^{2}$ & $\mu_{s}$ & $w$ & $\operatorname{PRE}\left(\hat{\mu}_{x(S G)}, \hat{\mu}_{x(E H)}\right)$ & $\operatorname{PRE}\left(\hat{\mu}_{x(S G)}, \hat{\mu}_{x(G)}\right)$ \\
\hline \multirow{4}{*}{5} & \multirow{4}{*}{0.5} & 0.2 & 451.84 & 34.03 \\
\hline & & 0.4 & 240.39 & 29.80 \\
\hline & & 0.6 & 163.76 & 28.27 \\
\hline & & 0.8 & 124.17 & 27.48 \\
\hline \multirow{4}{*}{5} & \multirow{4}{*}{1} & 0.2 & 358.48 & 100.00 \\
\hline & & 0.4 & 217.76 & 100.00 \\
\hline & & 0.6 & 156.38 & 100.00 \\
\hline & & 0.8 & 121.99 & 100.00 \\
\hline \multirow{4}{*}{5} & \multirow{4}{*}{1.5} & 0.2 & 279.22 & 156.01 \\
\hline & & 0.4 & 192.82 & 177.35 \\
\hline & & 0.6 & 147.26 & 188.61 \\
\hline & & 0.8 & 119.11 & 195.57 \\
\hline \multirow{4}{*}{5} & \multirow{4}{*}{2} & 0.2 & 225.39 & 194.04 \\
\hline & & 0.4 & 171.60 & 243.20 \\
\hline & & 0.6 & 138.54 & 273.41 \\
\hline & & 0.8 & 116.15 & 293.86 \\
\hline \multirow{4}{*}{5} & \multirow{4}{*}{2.5} & 0.2 & 190.46 & 218.73 \\
\hline & & 0.4 & 155.33 & 293.66 \\
\hline & & 0.6 & 131.14 & 345.25 \\
\hline & & 0.8 & 113.47 & 382.94 \\
\hline \multirow{4}{*}{5} & \multirow{4}{*}{3} & 0.2 & 167.48 & 234.96 \\
\hline & & 0.4 & 143.31 & 330.96 \\
\hline & & 0.6 & 125.23 & 402.74 \\
\hline & & 0.8 & 111.20 & 458.44 \\
\hline \multirow{4}{*}{10} & \multirow{4}{*}{0.5} & 0.2 & 474.38 & 29.80 \\
\hline & & 0.4 & 245.04 & 27.48 \\
\hline & & 0.6 & 165.18 & 26.67 \\
\hline & & 0.8 & 124.58 & 26.26 \\
\hline \multirow{4}{*}{10} & \multirow{4}{*}{1} & 0.2 & 414.03 & 100.00 \\
\hline & & 0.4 & 231.94 & 100.00 \\
\hline & & 0.6 & 161.09 & 100.00 \\
\hline & & 0.8 & 123.40 & 100.00 \\
\hline \multirow{4}{*}{10} & \multirow{4}{*}{1.5} & 0.2 & 347.53 & 177.35 \\
\hline & & 0.4 & 214.68 & 195.57 \\
\hline & & 0.6 & 155.31 & 203.71 \\
\hline & & 0.8 & 121.66 & 208.32 \\
\hline \multirow{4}{*}{10} & & 0.2 & 290.93 & 243.20 \\
\hline & 2 & 0.4 & 196.93 & 293.86 \\
\hline & 2 & 0.6 & 148.84 & 319.78 \\
\hline & & 0.8 & 119.63 & 335.52 \\
\hline & & 0.2 & 247.55 & 293.66 \\
\hline 10 & 25 & 0.4 & 180.84 & 382.94 \\
\hline 10 & 2.3 & 0.6 & 142.45 & 434.33 \\
\hline & & 0.8 & 117.51 & 467.71 \\
\hline & & 0.2 & 215.48 & 330.96 \\
\hline 10 & 3 & 0.4 & 167.21 & 458.44 \\
\hline 10 & 3 & 0.6 & 136.60 & 539.25 \\
\hline & & 0.8 & 115.47 & 595.06 \\
\hline & & 0.2 & 482.55 & 28.27 \\
\hline 15 & 05 & 0.4 & 246.65 & 26.67 \\
\hline 15 & 0.5 & 0.6 & 165.67 & 26.12 \\
\hline & & 0.8 & 124.72 & 25.85 \\
\hline
\end{tabular}




\begin{tabular}{|c|c|c|c|c|}
\hline \multicolumn{5}{|c|}{$C_{\gamma}=1.1$} \\
\hline$\gamma_{s}^{2}$ & $\mu_{s}$ & $w$ & $\operatorname{PRE}\left(\hat{\mu}_{x(S G)}, \hat{\mu}_{x(E H)}\right)$ & $\operatorname{PRE}\left(\hat{\mu}_{x(S G)}, \hat{\mu}_{x(G)}\right)$ \\
\hline \multirow{4}{*}{15} & \multirow{4}{*}{1} & 0.2 & 438.27 & 100.00 \\
\hline & & 0.4 & 237.46 & 100.00 \\
\hline & & 0.6 & 162.84 & 100.00 \\
\hline & & 0.8 & 123.91 & 100.00 \\
\hline \multirow{4}{*}{15} & \multirow{4}{*}{1.5} & 0.2 & 383.56 & 188.61 \\
\hline & & 0.4 & 224.45 & 203.71 \\
\hline & & 0.6 & 158.64 & 209.95 \\
\hline & & 0.8 & 122.67 & 213.36 \\
\hline \multirow{4}{*}{15} & \multirow{4}{*}{2} & 0.2 & 331.21 & 273.41 \\
\hline & & 0.4 & 209.89 & 319.78 \\
\hline & & 0.6 & 153.62 & 341.29 \\
\hline & & 0.8 & 121.14 & 353.70 \\
\hline \multirow{4}{*}{15} & \multirow{4}{*}{2.5} & 0.2 & 286.86 & 345.25 \\
\hline & & 0.4 & 195.52 & 434.33 \\
\hline & & 0.6 & 148.30 & 480.38 \\
\hline & & 0.8 & 119.45 & 508.51 \\
\hline \multirow{4}{*}{15} & \multirow{4}{*}{3} & 0.2 & 251.37 & 402.74 \\
\hline & & 0.4 & 182.36 & 539.25 \\
\hline & & 0.6 & 143.08 & 616.96 \\
\hline & & 0.8 & 117.72 & 667.12 \\
\hline \multirow{4}{*}{20} & \multirow{4}{*}{0.5} & 0.2 & 486.77 & 27.48 \\
\hline & & 0.4 & 247.48 & 26.26 \\
\hline & & 0.6 & 165.91 & 25.85 \\
\hline & & 0.8 & 124.79 & 25.64 \\
\hline \multirow{4}{*}{20} & \multirow{4}{*}{1} & 0.2 & 451.84 & 100.00 \\
\hline & & 0.4 & 240.39 & 100.00 \\
\hline & & 0.6 & 163.76 & 100.00 \\
\hline & & 0.8 & 124.17 & 100.00 \\
\hline \multirow{4}{*}{20} & \multirow{4}{*}{1.5} & 0.2 & 405.82 & 195.57 \\
\hline & & 0.4 & 229.98 & 208.32 \\
\hline & & 0.6 & 160.46 & 213.36 \\
\hline & & 0.8 & 123.21 & 216.06 \\
\hline \multirow{4}{*}{20} & \multirow{4}{*}{2} & 0.2 & 358.48 & 293.86 \\
\hline & & 0.4 & 217.76 & 335.52 \\
\hline & & 0.6 & 156.38 & 353.70 \\
\hline & & 0.8 & 121.99 & 363.88 \\
\hline \multirow{4}{*}{20} & \multirow{4}{*}{2.5} & 0.2 & 315.58 & 382.94 \\
\hline & & 0.4 & 205.06 & 467.71 \\
\hline & & 0.6 & 151.87 & 508.51 \\
\hline & & 0.8 & 120.60 & 532.50 \\
\hline \multirow{4}{*}{20} & \multirow{4}{*}{3} & 0.2 & 279.22 & 458.44 \\
\hline & & 0.4 & 192.82 & 595.06 \\
\hline & & 0.6 & 147.26 & 667.12 \\
\hline & & 0.8 & 119.11 & 711.63 \\
\hline
\end{tabular}

Table 5.1 Continued 


\begin{tabular}{|c|c|c|c|c|}
\hline \multicolumn{5}{|c|}{$C_{\gamma}=1.3$} \\
\hline$\gamma_{s}^{2}$ & $\mu_{s}$ & $w$ & $\operatorname{PRE}\left(\hat{\mu}_{x(S G)}, \hat{\mu}_{x(E H)}\right)$ & $\operatorname{PRE}\left(\hat{\mu}_{x(S G)}, \hat{\mu}_{x(G)}\right)$ \\
\hline \multirow{4}{*}{5} & \multirow{4}{*}{0.5} & 0.2 & 445.70 & 35.18 \\
\hline & & 0.4 & 239.08 & 30.46 \\
\hline & & 0.6 & 163.35 & 28.73 \\
\hline & & 0.8 & 124.06 & 27.83 \\
\hline \multirow{4}{*}{5} & \multirow{4}{*}{1} & 0.2 & 345.66 & 100.00 \\
\hline & & 0.4 & 214.14 & 100.00 \\
\hline & & 0.6 & 155.12 & 100.00 \\
\hline & & 0.8 & 121.61 & 100.00 \\
\hline \multirow{4}{*}{5} & \multirow{4}{*}{1.5} & 0.2 & 265.73 & 151.79 \\
\hline & & 0.4 & 187.88 & 173.24 \\
\hline & & 0.6 & 145.31 & 184.97 \\
\hline & & 0.8 & 118.47 & 192.36 \\
\hline \multirow{4}{*}{5} & \multirow{4}{*}{2} & 0.2 & 213.86 & 185.40 \\
\hline & & 0.4 & 166.47 & 232.95 \\
\hline & & 0.6 & 136.28 & 263.25 \\
\hline & & 0.8 & 115.35 & 284.25 \\
\hline \multirow{4}{*}{5} & \multirow{4}{*}{2.5} & 0.2 & 181.19 & 206.56 \\
\hline & & 0.4 & 150.62 & 277.17 \\
\hline & & 0.6 & 128.87 & 327.38 \\
\hline & & 0.8 & 112.62 & 364.93 \\
\hline \multirow{4}{*}{5} & \multirow{4}{*}{3} & 0.2 & 160.11 & 220.22 \\
\hline & & 0.4 & 139.19 & 309.03 \\
\hline & & 0.6 & 123.11 & 377.32 \\
\hline & & 0.8 & 110.36 & 431.46 \\
\hline \multirow{4}{*}{10} & \multirow{4}{*}{0.5} & 0.2 & 470.87 & 30.46 \\
\hline & & 0.4 & 244.33 & 27.83 \\
\hline & & 0.6 & 164.97 & 26.91 \\
\hline & & 0.8 & 124.52 & 26.44 \\
\hline \multirow{4}{*}{10} & \multirow{4}{*}{1} & 0.2 & 404.38 & 100.00 \\
\hline & & 0.4 & 229.64 & 100.00 \\
\hline & & 0.6 & 160.35 & 100.00 \\
\hline & & 0.8 & 123.18 & 100.00 \\
\hline \multirow{4}{*}{10} & \multirow{4}{*}{1.5} & 0.2 & 334.36 & 173.24 \\
\hline & & 0.4 & 210.83 & 192.36 \\
\hline & & 0.6 & 153.96 & 201.17 \\
\hline & & 0.8 & 121.25 & 206.23 \\
\hline \multirow{4}{*}{10} & & 0.2 & 277.27 & 232.95 \\
\hline & 2 & 0.4 & 192.12 & 284.25 \\
\hline & 2 & 0.6 & 146.99 & 311.44 \\
\hline & & 0.8 & 119.02 & 328.29 \\
\hline & & 0.2 & 234.99 & 277.17 \\
\hline 10 & 25 & 0.4 & 175.69 & 364.93 \\
\hline 10 & 2.3 & 0.6 & 140.30 & 417.33 \\
\hline & & 0.8 & 116.77 & 452.15 \\
\hline & & 0.2 & 204.52 & 309.03 \\
\hline 10 & 3 & 0.4 & 162.15 & 431.46 \\
\hline 10 & 3 & 0.6 & 134.32 & 511.87 \\
\hline & & 0.8 & 114.65 & 568.72 \\
\hline & & 0.2 & 480.10 & 28.73 \\
\hline 15 & 05 & 0.4 & 246.17 & 26.91 \\
\hline 15 & 0.5 & 0.6 & 165.52 & 26.29 \\
\hline & & 0.8 & 124.68 & 25.97 \\
\hline
\end{tabular}




\begin{tabular}{|c|c|c|c|c|}
\hline \multicolumn{5}{|c|}{$C_{\gamma}=1.3$} \\
\hline$\gamma_{s}^{2}$ & $\mu_{s}$ & $w$ & $\operatorname{PRE}\left(\hat{\mu}_{x(S G)}, \hat{\mu}_{x(E H)}\right)$ & $\operatorname{PRE}\left(\hat{\mu}_{x(S G)}, \hat{\mu}_{x(G)}\right)$ \\
\hline \multirow{4}{*}{15} & \multirow{4}{*}{1} & 0.2 & 430.74 & 100.00 \\
\hline & & 0.4 & 235.78 & 100.00 \\
\hline & & 0.6 & 162.32 & 100.00 \\
\hline & & 0.8 & 123.76 & 100.00 \\
\hline \multirow{4}{*}{15} & \multirow{4}{*}{1.5} & 0.2 & 371.89 & 184.97 \\
\hline & & 0.4 & 221.40 & 201.17 \\
\hline & & 0.6 & 157.62 & 208.03 \\
\hline & & 0.8 & 122.37 & 211.83 \\
\hline \multirow{4}{*}{15} & \multirow{4}{*}{2} & 0.2 & 317.67 & 263.25 \\
\hline & & 0.4 & 205.72 & 311.44 \\
\hline & & 0.6 & 152.11 & 334.52 \\
\hline & & 0.8 & 120.67 & 348.05 \\
\hline \multirow{4}{*}{15} & \multirow{4}{*}{2.5} & 0.2 & 273.25 & 327.38 \\
\hline & & 0.4 & 190.67 & 417.33 \\
\hline & & 0.6 & 146.42 & 465.53 \\
\hline & & 0.8 & 118.84 & 495.57 \\
\hline \multirow{4}{*}{15} & \multirow{4}{*}{3} & 0.2 & 238.66 & 377.32 \\
\hline & & 0.4 & 177.22 & 511.87 \\
\hline & & 0.6 & 140.94 & 591.32 \\
\hline & & 0.8 & 116.99 & 643.78 \\
\hline \multirow{4}{*}{20} & \multirow{4}{*}{0.5} & 0.2 & 484.89 & 27.83 \\
\hline & & 0.4 & 247.11 & 26.44 \\
\hline & & 0.6 & 165.81 & 25.97 \\
\hline & & 0.8 & 124.76 & 25.73 \\
\hline \multirow{4}{*}{20} & \multirow{4}{*}{1} & 0.2 & 445.70 & 100.00 \\
\hline & & 0.4 & 239.08 & 100.00 \\
\hline & & 0.6 & 163.35 & 100.00 \\
\hline & & 0.8 & 124.06 & 100.00 \\
\hline \multirow{4}{*}{20} & \multirow{4}{*}{1.5} & 0.2 & 395.55 & 192.36 \\
\hline & & 0.4 & 227.48 & 206.23 \\
\hline & & 0.6 & 159.64 & 211.83 \\
\hline & & 0.8 & 122.97 & 214.85 \\
\hline \multirow{4}{*}{20} & \multirow{4}{*}{2} & 0.2 & 345.66 & 284.25 \\
\hline & & 0.4 & 214.14 & 328.29 \\
\hline & & 0.6 & 155.12 & 348.05 \\
\hline & & 0.8 & 121.61 & 359.28 \\
\hline \multirow{4}{*}{20} & \multirow{4}{*}{2.5} & 0.2 & 301.85 & 364.93 \\
\hline & & 0.4 & 200.62 & 452.15 \\
\hline & & 0.6 & 150.23 & 495.57 \\
\hline & & 0.8 & 120.07 & 521.55 \\
\hline \multirow{4}{*}{20} & \multirow{4}{*}{3} & 0.2 & 265.73 & 431.46 \\
\hline & & 0.4 & 187.88 & 568.72 \\
\hline & & 0.6 & 145.31 & 643.78 \\
\hline & & 0.8 & 118.47 & 691.11 \\
\hline
\end{tabular}

Table 5.1 Continued 


\begin{tabular}{|c|c|c|c|c|}
\hline \multicolumn{5}{|c|}{$C_{\gamma}=1.5$} \\
\hline$\gamma_{s}^{2}$ & $\mu_{s}$ & $w$ & $\operatorname{PRE}\left(\hat{\mu}_{x(S G)}, \hat{\mu}_{x(E H)}\right)$ & $\operatorname{PRE}\left(\hat{\mu}_{x(S G)}, \hat{\mu}_{x(G)}\right)$ \\
\hline \multirow{4}{*}{5} & \multirow{4}{*}{0.5} & 0.2 & 440.98 & 36.07 \\
\hline & & 0.4 & 238.05 & 30.97 \\
\hline & & 0.6 & 163.03 & 29.09 \\
\hline & & 0.8 & 123.96 & 28.11 \\
\hline \multirow{4}{*}{5} & \multirow{4}{*}{1} & 0.2 & 336.36 & 100.00 \\
\hline & & 0.4 & 211.43 & 100.00 \\
\hline & & 0.6 & 154.17 & 100.00 \\
\hline & & 0.8 & 121.31 & 100.00 \\
\hline \multirow{4}{*}{5} & \multirow{4}{*}{1.5} & 0.2 & 256.39 & 148.87 \\
\hline & & 0.4 & 184.32 & 170.27 \\
\hline & & 0.6 & 143.88 & 182.28 \\
\hline & & 0.8 & 117.99 & 189.97 \\
\hline \multirow{4}{*}{5} & \multirow{4}{*}{2} & 0.2 & 206.12 & 179.59 \\
\hline & & 0.4 & 162.90 & 225.81 \\
\hline & & 0.6 & 134.67 & 256.00 \\
\hline & & 0.8 & 114.77 & 277.27 \\
\hline \multirow{4}{*}{5} & \multirow{4}{*}{2.5} & 0.2 & 175.09 & 198.56 \\
\hline & & 0.4 & 147.42 & 265.96 \\
\hline & & 0.6 & 127.30 & 314.96 \\
\hline & & 0.8 & 112.01 & 352.19 \\
\hline \multirow{4}{*}{5} & \multirow{4}{*}{3} & 0.2 & 155.32 & 210.64 \\
\hline & & 0.4 & 136.45 & 294.39 \\
\hline & & 0.6 & 121.67 & 360.00 \\
\hline & & 0.8 & 109.77 & 412.78 \\
\hline \multirow{4}{*}{10} & \multirow{4}{*}{0.5} & 0.2 & 468.14 & 30.97 \\
\hline & & 0.4 & 243.78 & 28.11 \\
\hline & & 0.6 & 164.80 & 27.10 \\
\hline & & 0.8 & 124.47 & 26.59 \\
\hline \multirow{4}{*}{10} & \multirow{4}{*}{1} & 0.2 & 397.14 & 100.00 \\
\hline & & 0.4 & 227.87 & 100.00 \\
\hline & & 0.6 & 159.77 & 100.00 \\
\hline & & 0.8 & 123.01 & 100.00 \\
\hline \multirow{4}{*}{10} & \multirow{4}{*}{1.5} & 0.2 & 324.86 & 170.27 \\
\hline & & 0.4 & 207.96 & 189.97 \\
\hline & & 0.6 & 152.93 & 199.24 \\
\hline & & 0.8 & 120.93 & 204.63 \\
\hline \multirow{4}{*}{10} & & 0.2 & 267.74 & 225.81 \\
\hline & ? & 0.4 & 188.64 & 277.27 \\
\hline & 2 & 0.6 & 145.61 & 305.26 \\
\hline & & 0.8 & 118.57 & 322.86 \\
\hline & & 0.2 & 226.44 & 265.96 \\
\hline 10 & 25 & 0.4 & 172.06 & 352.19 \\
\hline 10 & 2.3 & 0.6 & 138.73 & 405.03 \\
\hline & & 0.8 & 116.22 & 440.72 \\
\hline & & 0.2 & 197.20 & 294.39 \\
\hline 10 & 3 & 0.4 & 158.65 & 412.78 \\
\hline 10 & 3 & 0.6 & 132.70 & 492.45 \\
\hline & & 0.8 & 114.05 & 549.73 \\
\hline & & 0.2 & 478.18 & 29.09 \\
\hline & & 0.4 & 245.79 & 27.10 \\
\hline 15 & 0.5 & 0.6 & 165.41 & 26.42 \\
\hline & & 0.8 & 124.64 & 26.07 \\
\hline
\end{tabular}




\begin{tabular}{|c|c|c|c|c|}
\hline \multicolumn{5}{|c|}{$C_{\gamma}=1.5$} \\
\hline$\gamma_{s}^{2}$ & $\mu_{s}$ & $w$ & $\operatorname{PRE}\left(\hat{\mu}_{x(S G)}, \hat{\mu}_{x(E H)}\right)$ & $\operatorname{PRE}\left(\hat{\mu}_{x(S G)}, \hat{\mu}_{x(G)}\right)$ \\
\hline \multirow{4}{*}{15} & \multirow{4}{*}{1} & 0.2 & 425.00 & 100.00 \\
\hline & & 0.4 & 234.48 & 100.00 \\
\hline & & 0.6 & 161.90 & 100.00 \\
\hline & & 0.8 & 123.64 & 100.00 \\
\hline \multirow{4}{*}{15} & \multirow{4}{*}{1.5} & 0.2 & 363.29 & 182.28 \\
\hline & & 0.4 & 219.08 & 199.24 \\
\hline & & 0.6 & 156.83 & 206.56 \\
\hline & & 0.8 & 122.13 & 210.64 \\
\hline \multirow{4}{*}{15} & \multirow{4}{*}{2} & 0.2 & 308.00 & 256.00 \\
\hline & & 0.4 & 202.63 & 305.26 \\
\hline & & 0.6 & 150.98 & 329.41 \\
\hline & & 0.8 & 120.31 & 343.75 \\
\hline \multirow{4}{*}{15} & \multirow{4}{*}{2.5} & 0.2 & 263.78 & 314.96 \\
\hline & & 0.4 & 187.15 & 405.03 \\
\hline & & 0.6 & 145.02 & 454.55 \\
\hline & & 0.8 & 118.37 & 485.87 \\
\hline \multirow{4}{*}{15} & \multirow{4}{*}{3} & 0.2 & 230.00 & 360.00 \\
\hline & & 0.4 & 173.58 & 492.45 \\
\hline & & 0.6 & 139.39 & 572.73 \\
\hline & & 0.8 & 116.46 & 626.58 \\
\hline \multirow{4}{*}{20} & \multirow{4}{*}{0.5} & 0.2 & 483.41 & 28.11 \\
\hline & & 0.4 & 246.82 & 26.59 \\
\hline & & 0.6 & 165.72 & 26.07 \\
\hline & & 0.8 & 124.73 & 25.80 \\
\hline \multirow{4}{*}{20} & \multirow{4}{*}{1} & 0.2 & 440.98 & 100.00 \\
\hline & & 0.4 & 238.05 & 100.00 \\
\hline & & 0.6 & 163.03 & 100.00 \\
\hline & & 0.8 & 123.96 & 100.00 \\
\hline \multirow{4}{*}{20} & \multirow{4}{*}{1.5} & 0.2 & 387.89 & 189.97 \\
\hline & & 0.4 & 225.55 & 204.63 \\
\hline & & 0.6 & 159.01 & 210.64 \\
\hline & & 0.8 & 122.78 & 213.91 \\
\hline \multirow{4}{*}{20} & \multirow{4}{*}{2} & 0.2 & 336.36 & 277.27 \\
\hline & & 0.4 & 211.43 & 322.86 \\
\hline & & 0.6 & 154.17 & 343.75 \\
\hline & & 0.8 & 121.31 & 355.74 \\
\hline \multirow{4}{*}{20} & \multirow{4}{*}{2.5} & 0.2 & 292.15 & 352.19 \\
\hline & & 0.4 & 197.35 & 440.72 \\
\hline & & 0.6 & 149.00 & 485.87 \\
\hline & & 0.8 & 119.68 & 513.25 \\
\hline \multirow{4}{*}{20} & \multirow{4}{*}{3} & 0.2 & 256.39 & 412.78 \\
\hline & & 0.4 & 184.32 & 549.73 \\
\hline & & 0.6 & 143.88 & 626.58 \\
\hline & & 0.8 & 117.99 & 675.78 \\
\hline
\end{tabular}


We discuss Table 5.1 as follows:

(i) In general, the percent relative efficiency of the proposed estimator $\hat{\mu}_{x(S G)}$ with respect to Eichhorn and Hayre's (1983) estimator $\hat{\mu}_{x(E H)}$ is larger than $100 \%$. It follows that the proposed estimator $\hat{\mu}_{x(S G)}$ is always better than the Eicchorn and Hayre's (1983) estimator $\hat{\mu}_{x(E H)}$. Thus the proposed randomized response model is always superior to the Eichhorn and Hayre's (1983) randomized response model.

(ii) The proposed estimator $\hat{\mu}_{x(S G)}$ is: (a) inferior (b) equally efficient, and (c) always superior; to the Gupta et al's (2002) estimator $\hat{\mu}_{x(G)}$ when $\mu_{s}<1, \mu_{s}=1$ and $\mu_{s}>1$ respectively.

(iii) For different values of $\left(\gamma_{s}^{2}, \mu_{s}\right)$, the percent relative efficiency of $\hat{\mu}_{x(S G)}$ with respect to $\hat{\mu}_{x(E H)}$ decreases as the value of $w$ increases, whereas the percent relative efficiency of $\hat{\mu}_{x(S G)}$ with respect to $\hat{\mu}_{x(G)}$ increases with the increase in the value of $w$. Thus the proposed randomized response model works better than Gupta et al's (2002) randomized response model for higher values of sensitivity level $w$ and $\mu_{s}>1$.

(iv) It is also observed that the values of $\operatorname{PRE}\left(\hat{\mu}_{x(S G)}, \hat{\mu}_{x(E H)}\right)$ and $\operatorname{PRE}\left(\hat{\mu}_{x(S G)}, \hat{\mu}_{x(G)}\right)$ decrease with increasing values of the coefficient of variation $C_{\gamma}$.

(v) A larger gain in efficiency is observed by using the proposed estimator $\hat{\mu}_{x(S G)}$ over Gupta et al's (2002) estimator $\hat{\mu}_{x(G)}$ is observed for the values $\left(\mu_{s}>1\right)$. We can also perceive a loss in efficiency for the values $\left(\mu_{s}<1\right)$.

It is further observed from Table 5.1 that the percent relative efficiency of the suggested estimator $\hat{\mu}_{x(S G)}$ with respect to Eichhorn and Hayre's (1983) estimator 
$\hat{\mu}_{x(E H)}$ and Gupta et al's (2002) estimator $\hat{\mu}_{x(G)}$ are larger than $100 \%$ for the selected parametric values [i.e $\left(\gamma_{s}^{2}, C_{x}, w\right)$ and $\left.\left(\mu_{s} \geq 1\right)\right]$ as given in Table 5.1. It follows that the proposed randomized response model is superior to the Eichhorn and Hayre's and Gupta et al's (2002) randomized response model for $\mu_{s}>1$.

\section{CONCLUDING REMARK}

The proposed method has the advantage of being able to simultaneously estimate both the average response and the sensitivity level of sensitive survey questions. While comparing this method with other methods, one should keep in mind that the proposed estimator estimates for all values of $\mu_{s}$ but have a smaller variance than Gupta et al's (2002) estimator $\hat{\mu}_{x(G)}$ for $\mu_{s}>1$. We have also developed the procedure for estimating the sensitivity level $w$.

\section{REFERENCES}

Bhargava, M. and Singh, R. (2001): Efficiency Comparison of Certain Randomized Response Schemes with U-Model. Journal of The Indian Society of Agricultural Statistics, 54(1),19-28.

Bhargava, M. and Singh, R. (2002): On The Efficiency Comparison Of Certain Randomized Response Strategies. Metrika, 55(3), 191-197.

Eichhorn, B. H., and Hayre, L. S. (1983): Scrambled Randomized Response Methods For Obtaining Sensitive Quantitative Data. Journal of Statistical Planning and Inference, 7,307-316.

Gjestvang, C. R., and Singh, S. (2006): A New Randomized Response Model. Journal of The Royal Statistical Society B, 68,523-530.

Gupta, S.N., Gupta, B.C. and Singh, S. (2002): Estimation of Sensitivity Level of Personal Interview Survey Questions. Journal of Statistical Planning and Inference, 100, 239-247.

Singh, H.P., and Mathur, N. (2003): Modified Optional Randomized Response Sampling Techniques. Journal of Indian Society of Agriculture and Statistics 56(2), 199-206.

Singh, H.P., and Tarray, T.A. (2014): A Stratified Mangat and Singh's Optional Randomized Response Model Using Proportional and Optimal Allocation. Statistica,73(1), 65-83.

Singh S, and Joarder A.H. (1997): Optional Randomized Response Technique for Sensitive Quantitative Variable. Metron 15:151-157

Tarray, T.A., Singh, H.P. and Yan, Z. (2015): A Dexterous Optional Randomized Response Model. Sociological Methods and Research. Doi:10.1177/0049124115605332

Tarray, T.A., and Singh, H.P. (2017): An Optional Randomized Response Model for Estimating a Rare Sensitive Attribute Using Poisson Distribution. Communications in Statistics-Theory and Methods. 46(6), 2638-2654. 
Tracy, D.S., and Mangat, N.S., (1996): Some developments in randomized response sampling during the last decade-A follow up of review by Chaudhuri and Mukherjee. Journal of Applied Statistical Sciences. 4 (2), 147-158.

Warner, S.L. (1965): Randomized Response: A Survey Technique For Eliminating Evasive Answer Bias. Journal of American Statistical Association, 60, 63-69.

Zou, G. (1997): Two-Stage Randomized Response Procedures as Single Stage Procedures. Australian Journal of Statistics, 39,235-236.

Housila P. Singh

School of Studies in Statistics

Vikram University

Ujjain, M.P., India

Email: hpsujn@gmail.com

Swarangi M. Gorey

School of Studies in Statistics

Vikram University

Ujjain, M.P., India

Email: swarangi.gorey@gmail.com 